\title{
64-Slice spiral double-low CT to evaluate the degree of stenosis and plaque composition in diagnosing coronary artery disease
}

\author{
XIAO-RONG FENG ${ }^{1}$, LI-HUA LIANG ${ }^{1}$, JU-FANG WU ${ }^{1}$, YAO-QIANG CHEN $^{1}$ and SHUANG LIANG ${ }^{2}$ \\ Departments of ${ }^{1}$ Imaging and ${ }^{2}$ Ultrasonography, The Eighth Affiliated Hospital, \\ Sun Yat-sen University, Shenzhen, Guangdong 518033, P.R. China
}

Received March 13, 2017; Accepted June 22, 2017

DOI: $10.3892 /$ etm.2017.4890

\begin{abstract}
This study examined the application of 64-slice spiral double-low computed tomography (CT) to evaluate the degree of coronary artery stenosis. We examined 45 patients with coronary heart disease by 64-slice spiral double-low CT and coronary angiography (CAG) to determine CT accuracy in evaluating coronary artery stenosis. Imaging analysis from 64-slice spiral double-low CT identified 199 segments with coronary stenosis from 45 patients, including 46 segments with mild stenosis, 38 with moderate stenosis and 115 with severe stenosis or artery occlusion. CT analysis agreed with CAG on the identification of the degree of stenosis in 122 segments, with an overall accuracy of $61.3 \%$. The accuracy for serious stenosis or occlusion was the highest at $69.6 \%$. We also found a strong correlation between coronary plaque compositions and the degree of stenosis. Correspondence analysis showed that the presence of soft plaques closely correlated with severe stenosis, whereas mixed plaques closely correlated with moderate stenosis. Overall, 64-slice spiral double-low CT imaging can effectively assess the degree of coronary artery stenosis in patients with coronary heart disease and accurately detect plaque composition. Thus, 64-slice spiral double-low $\mathrm{CT}$ imaging can predict the risk of coronary heart disease and the degree of coronary artery stenosis, which is helpful for early diagnosis and treatment of coronary heart disease.
\end{abstract}

\section{Introduction}

Coronary heart disease is a common condition caused mainly by the imbalance between the blood supply from the coronary artery and the myocardial needs (1). In recent years, the incidence and fatality rate of coronary heart diseases in China have increased. Coronary heart disease seriously threatens the

Correspondence to: Dr Xiao-Rong Feng, Department of Imaging, The Eighth Affiliated Hospital, Sun Yat-sen University, 3025 Shennan Middle Road, Futian, Shenzhen, Guangdong 518033, P.R. China E-mail: 37051008@qq.com

Key words: 64-slice spiral CT, coronary heart disease, coronary artery, stenosis degree, plaque composition physical and psychological health (2). Thus, effective prevention and treatment of coronary heart disease is an important topic. Vulnerable plaques can be observed at the early stage and the active stage of coronary arteriosclerosis $(3,4)$. Such plaques contain lipids and fibers. Therefore, early detection of vulnerable plaques is key for the prevention and treatment of coronary heart disease. At present, the golden standard for clinical diagnosis of the stability of arteriosclerosis plaques is intracoronary ultrasound. But this technology is limited by high cost and other factors in clinical application $(5,6)$. The 64-slice spiral double-low computed tomography (CT) (low-tube voltage, low-iodine flow rate) has high spatial and temporal resolution, offers satisfactory images, directly shows the degree of coronary arteriosclerosis, and effectively judges the stenosis degree of coronary artery and the compositions of plaques. This imaging technique can determine the composition of coronary plaques of coronary artery non-invasively. This study focused on the application of 64-slice spiral double-low CT for the evaluation of coronary artery stenosis and discuss the correlation between stenosis degree and plaque composition.

\section{Materials and methods}

General materials. We recruited 45 patients admitted in our hospital with coronary heart disease from February 2015 to December 2016. The cohort had 25 males and 20 females. Ages ranged from 41-65 years, averaging 57.25 \pm 6.51 years. Height range $155-173 \mathrm{~cm}$, averaging $165.32 \pm 4.27 \mathrm{~cm}$. Weight was $45-56 \mathrm{~kg}$, averaging $56.85 \pm 6.21 \mathrm{~kg}$. BMI was $19-23 \mathrm{~kg} / \mathrm{m}^{2}$, averaging $21.08 \pm 2.36 \mathrm{~kg} / \mathrm{m}^{2}$. Inclusion criteria: i) BMI, $20-25 \mathrm{~kg} / \mathrm{m}^{2}$; ii) heart rate lower than 70 beats/min, patients with high heart rate or arrhythmia received oral Betoloc to reduce heart rate; and iii) breath exercise required patients to hold breath for over 10 sec once and keep the same breathing movement. Exclusion criteria: i) Serious arrhythmia; ii) rheumatic heart diseases; iii) myocardial diseases; and iv) coronary artery stenting or coronary artery bypass grafting. This study was approved by the Ethics Committee of The Eighth Affiliated Hospital, Sun Yat-sen University. Signed written informed consents were obtained from the patients and/or guardians.

Equipment and devices. 64-Slice spiral double-low CT scanner (Somatom Sensation; Siemens, Munich, Germany), 
contrast media injector (Ulrich Medical, Ulm, Germany), contrast media, $370 \mathrm{mg} \mathrm{I} / \mathrm{ml}$ iopromide injection.

Detection methods. The tube voltage of the 64-slice spiral double-low CT scanner was set as $120 \mathrm{kV}$. Current quality was set according to patient BMI, generally to 500-650 mA. The collimation was set at $0.66 \mathrm{~mm}$, pitch 1.0-1.4, FOV $250 \mathrm{~mm}$ and matrix $512 \times 512$. We scanned the area $2 \mathrm{~cm}$ below the trachea carina of patients to the area $2 \mathrm{~cm}$ from the subphrenic point. First, the chest was scanned with the patients holding their breath. Then, the sequence scanning was completed to obtained images at the diastolic stage of cardiac cycle. The slice thickness was set as $3 \mathrm{~mm}$. Generally, 90 sections can be obtained scanning from ascending aorta to the cardiac apex.

Image output and reconstruction. After transferring images to the Siemens post-processing working station, MPR, VR, MIP and CPR were reconstructed to visualize the short axis of the coronary artery.

Measurement and evaluation of vascular stenosis by MSCT and coronary angiography (CAG). CCTA was used to measure vessel diameter of the areas with the worst stenosis. The average of normal diameter of coronary artery before and after stenosis was used to calculate the percentage of stenosis diameter/normal diameter. According to the lumen of the stenosis area, coronary stenosis was divided into 4 levels by CAG: i) no stenosis; ii) mild stenosis $(<50 \%)$; iii) moderate stenosis (50-75\%); and iv) serious stenosis or occlusion $(>75 \%)$.

Statistical analysis. SPSS 20.0 statistical software (IBM, New York, NY, USA) was applied for analysis. Measurement data were expressed as mean \pm standard deviation and tested by $\chi^{2}$ test. Enumeration data were expressed by independent sample t-test. $\chi^{2}$ test was used to analyze compositions of coronary plaques and stenosis degrees. Correspondence analysis was made to predict the correlation degree between the two variables. $\mathrm{P}<0.05$ was considered to indicate a statistically significant difference.

\section{Results}

Evaluable conditions of coronary artery images by MSCT. According to the piecewise method of coronary artery analysis by the American Heart Association, we obtained 632 coronary artery segments from 45 patients. Thirty-nine segments that could not be evaluated were excluded. Thus, we evaluated 593 coronary artery segments by MSCT, with an evaluable rate of $93.8 \%$ (Table I).

CAG diagnosis. We used CAG to evaluate 632 coronary artery segments from 45 patients. Of the 448 segments $70.9 \%$ showed no obvious stenosis, $42(6.6 \%)$ showed mild stenosis, $34(5.4 \%)$ showed moderate stenosis and 108 (17.1\%) showed serious stenosis (Table II).

Accuracy of coronary artery stenosis by 64-slice spiral double-low CT. Of the 632 segments analyzed, 199 segments from 45 patients showed vascular stenosis caused by plaques by 64-slice spiral double-low CT. Forty-six segments showed
Table I. Evaluation of lesions by 64-slice spiral double-low CT.

\begin{tabular}{lcc}
\hline Evaluation conditon & Segment no. & Percentage (\%) \\
\hline Evaluable & 593 & 93.8 \\
Unevaluable & 39 & 6.2 \\
Motion artifact & 18 & 2.8 \\
Vessel wall calcification & 7 & 1.1 \\
Thin lumen $(<1.5 \mathrm{~mm})$ & 9 & 1.4 \\
\hline
\end{tabular}

CT, computed tomography.

Table II. Lesions revealed by CAG.

\begin{tabular}{lcc}
\hline Evaluation condition & $\begin{array}{c}\text { No. of } \\
\text { segments }\end{array}$ & $\begin{array}{c}\text { Percentage } \\
(\%)\end{array}$ \\
\hline No stenosis & 448 & 70.9 \\
Mild stenosis $(<50 \%)$ & 42 & 6.6 \\
Moderate stenosis $(50-75 \%)$ & 34 & 5.4 \\
Serious stenosis or & 108 & 17.1 \\
occlusion $(>75 \%)$ & & \\
\hline
\end{tabular}

CAG, coronary angiography.

Table III. Coronary artery stenosis and plaque composition by 64-slice spiral double-low CT.

Coronary artery stenosis degree

\begin{tabular}{lcccr}
\cline { 2 - 4 } $\begin{array}{l}\text { Plaque } \\
\text { composition }\end{array}$ & $\begin{array}{c}\text { Mild } \\
\text { stenosis }\end{array}$ & $\begin{array}{c}\text { Moderate } \\
\text { stenosis }\end{array}$ & $\begin{array}{c}\text { Serious stenosis } \\
\text { or occlusion }\end{array}$ & Total \\
\hline Mixed & 2 & 2 & 5 & 9 \\
Soft & 4 & 2 & 9 & 15 \\
Hard & 1 & 1 & 1 & 3 \\
Total & 7 & 5 & 15 & 27
\end{tabular}

CT, computed tomography.

mild stenosis, 38 moderate stenosis and 115 serious stenosis or occlusion) (Fig. 1). Evaluation by 64-slice spiral double-low CT agreed with CAG on the evaluation of stenosis degree in 122 segments. The accuracy rate was $61.3 \%(122 / 199)$. The accuracy rate for mild, moderate and serious stenosis or occlusion was $47.8 \%$ (22/46), 52.6\% (20/38) and 69.6\% (80/115), respectively. The diagnostic accuracy was the highest for serious stenosis or occlusion.

Correlation between degree of coronary artery stenosis and plaque composition by 64-slice spiral double-low CT. Among the 45 patients examined, 18 showed no obvious stenosis, 7 mild stenosis, 5 moderate stenosis and 15 serious stenosis or occlusion. Fifteen cases presented with soft plaques, 3 with hard plaques and 9 with mixed plaques. Comparison 
A

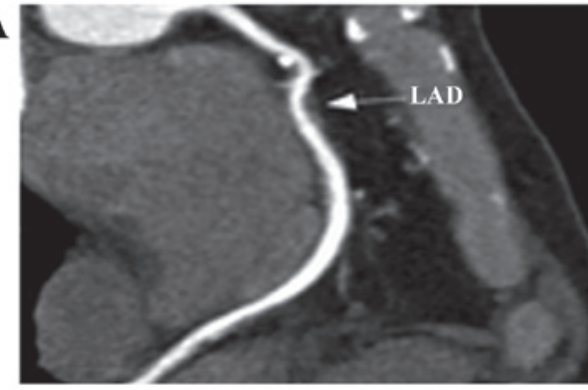

C

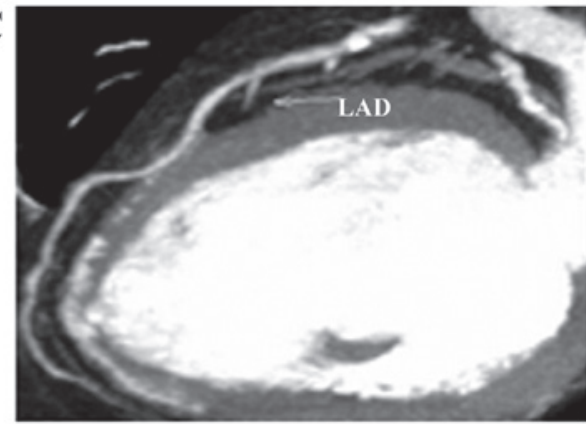

$\mathbf{E}$

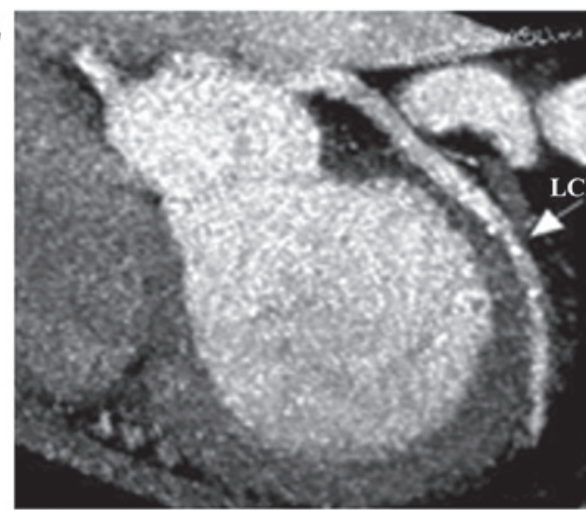

B

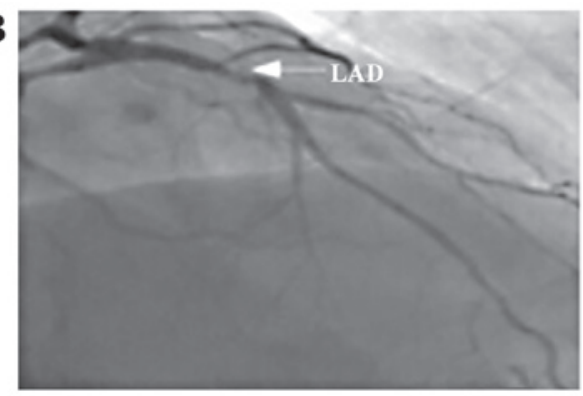

D

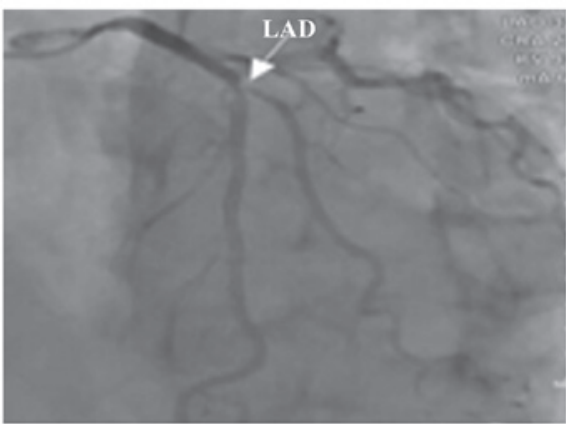

F

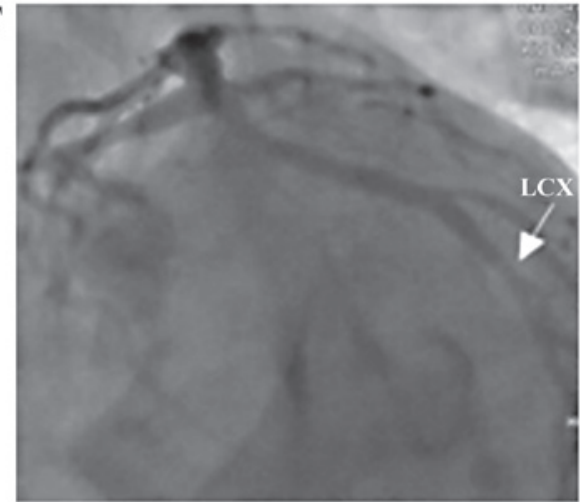

Figure 1. Evaluation of coronary artery stenosis by 64-slice spiral double-low CT. (A and B) Contrast of $60 \%$ stenosis in left anterior descending coronary near segment (moderate stenosis). (C and D) Contrast of $80 \%$ stenosis in left anterior descending coronary near segment (serious stenosis). (E and F) Contrast of $30 \%$ stenosis in left anterior descending coronary near segment (mild stenosis). CT, computed tomography.

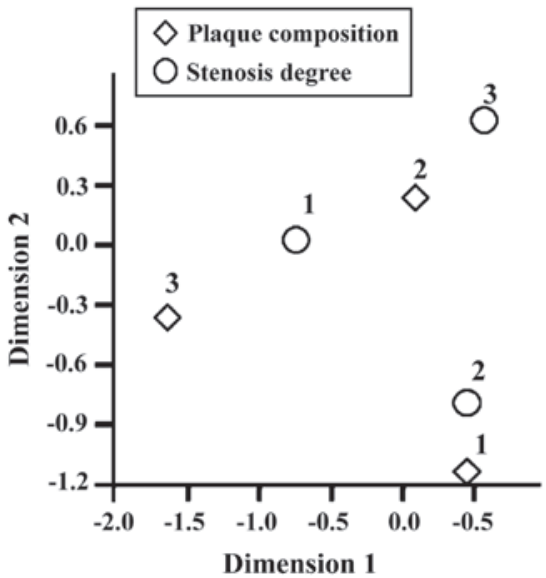

Figure 2. Correspondence analysis image of the correlation between coronary artery stenosis degree and plaque composition.

between soft and hard plaques revealed statistical significance, indicating that the degree of coronary artery stenosis was closely correlated with plaque composition (Table III) $\left(\chi^{2}=9.627, \mathrm{p}<0.05\right)$. Correspondence analysis indicated that soft plaques were closely correlated with serious stenosis and mixed plaques were closely correlated with moderate stenosis (Fig. 2). The composition of coronary plaques and stenosis is shown in Fig. 3.

\section{Discussion}

Previous research shows that hemodynamic changes can only be identified when the coronary artery stenosis is $\geq 50 \%$ (7-9). Thus, evaluation of coronary artery by MSCT mainly takes $50 \%$ stenosis as the critical point. However, the clinical significance varies with the degree of coronary artery stenosis (10-12). When coronary artery stenosis is $\geq 70 \%$, stent intervention is recommended. At the early stage of coronary heart disease, the coronary artery stenosis degree is typically mild. But patients at this stage show no obvious blood flow changes. Clinically, mild coronary artery stenosis is hard to diagnose (13-15). Since the diameter of the coronary artery is small and is easily influenced by heart systole, patients are asked to interrupt the breath and avoid other motion while conducting conventional $\mathrm{CT}$ or magnetic resonance imaging (MRI) (16-18). To overcome the limitations of CT 
A

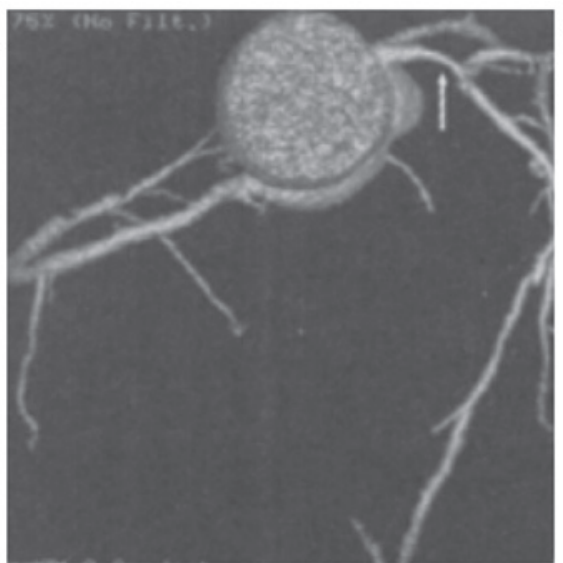

C

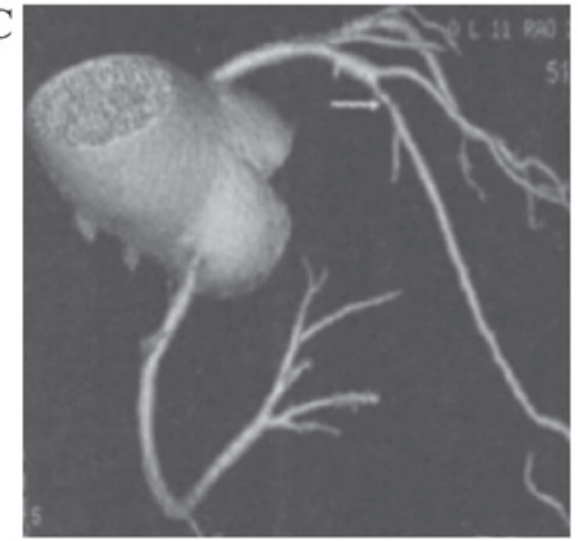

B

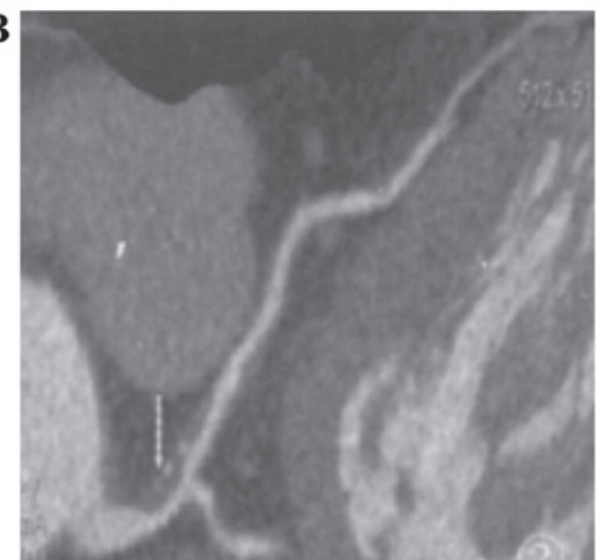

$\mathbf{D}$

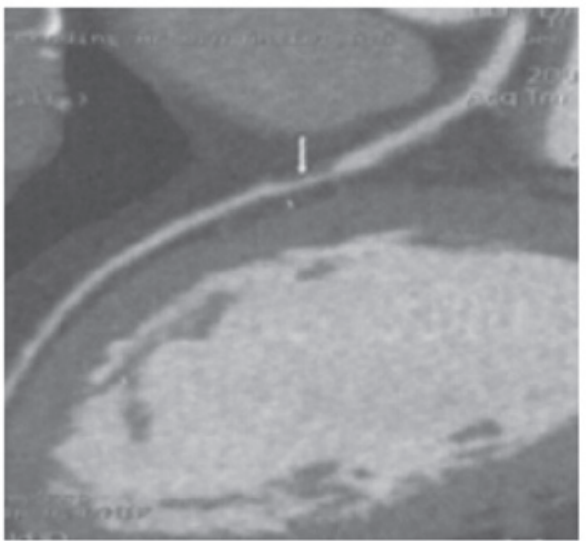

Figure 3. Coronary artery stenosis and plaque composition. (A) Heart VR reconstruction image shows obvious stenosis in the coronary arterial tree and the trunk lumen of the left coronary artery. (B) LAD CPR image shows formation of local soft plaques in the trunk of the left coronary artery and the lumen of LAD coronary near segment. Internal density is not even. There is small calcified shadow and obvious stenosis of local lumen. (C) Heart VR reconstruction image shows limited stenosis in coronary arterial tree and the lumen of the LAD middle segments. (D) LAD CPR image shows irregular soft plaques fillingdefect shadows in the lumen of LAD middle segments. The density is even and local lumen shows obvious stenosis.

and MRI, high resolution CT can be used to diagnose coronary artery stenosis. Compared with conventional CT, the scanning speed of 64-slice spiral double-low CT is substantially increased and can provide 3D construction images of the cardiovascular system. Additionally, this technology is non-invasive, safe, reliable, easy to operate and inexpensive. In recent years, it has attracted increasing attention in the diagnosis of coronary heart disease $(19,20)$.

Herein, we report the accuracy of 64-slice spiral double-low CT for the diagnosis of $<50 \%, 50 \%, \geq 70 \%$ coronary artery stenosis. 64-Slice spiral double-low CT agreed with CAG on the determination of stenosis degree of 122 segments with an overall accuracy of $61.3 \%$. The accuracy of serious stenosis or occlusion was the highest at $69.6 \%$. These results indicate that 64-slice spiral double-low CT can provide a relatively accurate diagnosis for the degree of coronary artery stenosis.

The pathologic evolution of coronary plaques and changes of coronary artery stenosis are both complex processes. Plaque composition can cause ulceration, bleeding and local lumen vessel reconstruction. Research has shown that serious coronary artery stenosis is mostly caused by soft plaques whereas moderate stenosis mainly by mixed plaques (21). The reason may be that the coronary artery is closely correlated with the pathologic evolution of plaques. Plaques at the early stage in the coronary artery are mainly lipid in composition, and normal lumen diameter can be maintained under the action of local vessel compensatory enlargement. However, as the lipid pool increases in the coronary artery, the lumen becomes narrower. Moreover, the inflammatory necrosis released by inflammatory reactions in the coronary artery can also induce quick increase of plaque size. Additionally, the lysozyme released by inflammatory cells will make the fibrous cap weaker and further induce ulceration and rupture of fibrous caps. Moreover, lysozyme can promote the formation of thrombus on the plaque surface and continue to worsen the stenosis of the coronary artery lumen. If the plaques show bleeding, rupture, and thrombus formation, more serious stenosis and occlusion will be induced. In addition, if plaques show calcification, local lumen can generate negative reconstruction, and lead to more serious stenosis of the lumen (22). Our results show correlation between coronary plaque composition and stenosis degree. Correspondence analysis indicated close correlation between soft plaque and serious stenosis, and close correlation between mixed plaques and moderate stenosis.

In conclusion, 64-slice spiral double-low CT can effectively evaluate the degree of coronary artery stenosis of patients with coronary heart disease and accurately detect plaque composition. Thus, 64-slice spiral double-low CT can predict the risk of coronary heart disease based on the degree of coronary artery stenosis. Some of the benefits include early detection, diagnosis and treatment of coronary heart disease. 


\section{Acknowledgements}

This study was supported in part by grants from Shenzhen Science and Technology Innovation Commission Project 2016 (no. 148).

\section{References}

1. Kishi S, Magalhães TA, Cerci RJ, Matheson MB, Vavere A, Tanami Y, Kitslaar PH, George RT, Brinker J, Miller JM, et al: Total coronary atherosclerotic plaque burden assessment by CT angiography for detecting obstructive coronary artery disease associated with myocardial perfusion abnormalities. J Cardiovasc Comput Tomogr 10: 121-127, 2016.

2. Zimmerman SL, Lin CT, Chu LC, Eng J and Fishman EK: Remote reading of coronary CTA exams using a tablet computer: Utility for stenosis assessment and identification of coronary anomalies. Emerg Radiol 23: 255-261, 2016.

3. Sun Z, Lin C, Davidson R, Dong C and Liao Y: Diagnostic value of 64-slice CT angiography in coronary artery disease: A systematic review. Eur J Radiol 67: 78-84, 2008.

4. Chen JW and Hsu RB: Impact of surgeon experience on the rate of blood transfusion in off-pump coronary artery bypass. J Formos Med Assoc 115: 145-151, 2016

5. Stuijfzand WJ, Danad I, Raijmakers PG, Marcu CB, Heymans MW, van Kuijk CC, van Rossum AC, Nieman K, Min JK, Leipsic J, et al: Additional value of transluminal attenuation gradient in $\mathrm{CT}$ angiography to predict hemodynamic significance of coronary artery stenosis. JACC Cardiovase Imaging 7: 374-386, 2014.

6. Marwan M, Hausleiter J, Abbara S, Hoffmann U, Becker C, Ovrehus K, Ropers D, Bathina R, Berman D, Anders K, et al: Multicenter evaluation of coronary dual-source CT angiography in patients with intermediate risk of coronary artery stenoses (MEDIC): Study design and rationale. J Cardiovasc Comput Tomogr 8: 183-188, 2014.

7. Hamdan A, Wellnhofer E, Konen E, Kelle S, Goitein O, Andrada B, Raanani E, Segev A, Barbash I, Klempfner R, et al: Coronary CT angiography for the detection of coronary artery stenosis in patients referred for transcatheter aortic valve replacement. J Cardiovasc Comput Tomogr 9: 31-41, 2015.

8. Hrusca A, Rachisan AL, Gach P, Pico H, Sorensen C, Bonello B, Ovaert C, Petit P, Fouilloux V, Mace L, et al: Detection of pulmonary and coronary artery anomalies in tetralogy of Fallot using non-ECG-gated CT angiography. Diagn Interv Imaging 97: 543-548, 2016

9. Park EA, Lee W, Sang JP, Kim YK and Hwang HY: Influence of coronary artery diameter on intracoronary transluminal attenuation gradient during CT angiography. JACC Cardiovasc Imaging 9: 1074-1083, 2016.

10. Leschka S, Stolzmann P and Alkadhi H: Recent developments in coronary computed tomography imaging. Imaging Med 1: 103-114, 2016.
11. Plank F, Burghard P, Friedrich G, Dichtl W, Mayr A, Klauser A, Wolf $\mathrm{F}$ and Feuchtner G: Quantitative coronary CT angiography: Absolute lumen sizing rather than \% stenosis predicts hemodynamically relevant stenosis. Eur Radiol 26: 3781-3789, 2016.

12. Ramanan RV and Ramalingam A: Coronary artery bypass graft failure and its relationship to target artery percentage stenosis and competitive flow. A CT angiographic analysis. Apollo Med 11: 245-254, 2014

13. Hwang JW, Kim SM, Park SJ, Cho EJ, Lee SC, Choe YH and Park SW: A preoperative assessment of significant coronary stenosis based on a semiquantitative analysis of coronary artery calcification on noncontrast computed tomography in aortic stenosis patients undergoing aortic valve replacement. Medicine (Baltimore) 95: e2906, 2016.

14. Sinha SK, Khanra D, Jha MJ, Singh K, Razi M, Goel A, Mishra V, Asif M, Sachan M, Afdaali N, et al: Unusual survival of anomalous left coronary artery from the pulmonary artery with severe rheumatic mitral stenosis in septuagenarian women: Foes becoming friends? J Clin Med Res 8: 760-763, 2016.

15. Obrenovic-Kircanski B, Panic D, Parapid B, Karan R, Kovacevic-Kostic N and Skoric-Hinic L: Role of risk factors in prediction of asymptomatic carotid artery stenosis in patients with coronary artery disease. Acta Med Mediter 32: 63-67, 2016.

16. Jin KN, De Cecco CN, Caruso D, Tesche C, Spandorfer A, Varga-Szemes A and Schoepf UJ: Myocardial perfusion imaging with dual energy CT. Eur J Radiol 85: 1914-1921, 2016.

17. Ascarelli A, Francone M, Cannata D, Cannavale A, Carbone I and Passariello R: Role of multidetector CT in the evaluation of coronary artery bypass grafts. Imaging Med 2: 77-86, 2010.

18. Psaltis PJ, Talman AH, Munnur K, Cameron JD, Ko BS, Meredith IT, Seneviratne SK and Wong DT: Relationship between epicardial fat and quantitative coronary artery plaque progression: Insights from computer tomography coronary angiography. Int J Cardiovasc Imaging 32: 317-328, 2016.

19. Patchett ND, Pawar S and Miller EJ: Visual identification of coronary calcifications on attenuation correction CT improves diagnostic accuracy of spect/ct myocardial perfusion imaging. J Nucl Cardiol 24: 711-720, 2017.

20. Orzan M, Jako B, Blendea C, Benedek A, Bajka B and Benedek I: Correlations between the gradient of contrast density, evaluated by cardio $\mathrm{CT}$, and functional significance of coronary artery stenosis. Acta Med Marisiensis 62: 199-201, 2016.

21. Dai N, Lv HJ, Xiang YF, Fan B, Li WM and Xu YW: Three-dimensional modeling and numerical analysis of fractional flow reserve in human coronary arteries. Postepy Kardiol Interwencyjnej 12: 25-31, 2016.

22. Shekarchi B, Motevalli M, Mohammadzadeh A and Noormohammadzadeh M: Diagnostic accuracy of dual-source CT angiography for evaluation of coronary artery and comparative analysis of the DSCT angiography of the internal carotid artery plaque with the histopathological specimens. Comp Clin Pathol 25: 7-14, 2016. 